\title{
The Case for a Quantum Theory on a Hilbert Space with an Inner Product of Indefinite Signature
}

\author{
Otto C. W. Kong \\ Department of Physics and Center for High Energy and High Field Physics, National Central University, Taiwan \\ Email: otto@phy.ncu.edu.tw
}

How to cite this paper: Kong, O.C.W. (2020) The Case for a Quantum Theory on a Hilbert Space with an Inner Product of Indefinite Signature. Journal of High Energy Physics, Gravitation and Cosmology, 6, 43-48.

https://doi.org/10.4236/jhepgc.2020.61005

Received: October 23, 2019

Accepted: December 7, 2019

Published: December 10, 2019

Copyright ( 2020 by author(s) and Scientific Research Publishing Inc. This work is licensed under the Creative Commons Attribution International License (CC BY 4.0).

http://creativecommons.org/licenses/by/4.0/

\section{Open Access}

\begin{abstract}
We present the theoretical considerations for the case of looking into a generalization of quantum theory corresponding to having an inner product with an indefinite signature on the Hilbert space. The latter is essentially a direct analog of having the Minkowski spacetime with an indefinite signature generalizing the metric geometry of the Newtonian space. In fact, the explicit physics setting we have in mind is exactly a Lorentz covariant formulation of quantum mechanics, which has been discussed in the literature for over half a century yet without a nice full picture. From the point of view of the Lorentz symmetry, indefiniteness of the norm for a Minkowski vector may be the exact correspondence of the indefiniteness of the norm for a quantum state vector on the relevant Hilbert space. That, of course, poses a challenge to the usual requirement of unitarity. The related issues will be addressed.
\end{abstract}

\section{Keywords}

Lorentz Covariant Quantum Theory, Pseudo-Unitary Representation

\section{Introduction}

Quantum physics with the superposition principle is to be realized with states depicted by vectors on a Hilbert space, a complex vector space, usually endowed with a sesqulinear inner product with a positive definite signature, i.e. giving a positive definite norm. A proper symmetry transformation has to preserve the inner product, hence to be unitary. The latter is of central importance to the standard probability interpretation. However, there has been important theoretical development on understanding quantum mechanics from a symmetry/ spacetime and symplecto-geometric perspective that can get around the proba- 
bility interpretation [1] [2]. After all, for the deterministic Schrödinger dynamics of a quantum system, there is no issue of probability. Measurement, von Neumann measurement, in particular, is a much more involved physical setting, especially more so for a Lorentz covariant quantum theory. The simple bottom line here is that even in the setting of quantum mechanics with the Copenhagen interpretation, the born probability picture should not be strictly required to be extended to a spacetime description. Maintaining the total probability of finding a particle somewhere in the space, at a particular moment of its existence, to be unity is one thing, asking for the total probability of finding a particle somewhere in spacetime to be unity is quite another. For a particle wavefunction, as a function of the Minkowski spacetime coordinates $x^{\mu}$ for example, it could be enough that a restriction of it to any particular time value admits the born picture description. Focusing on a formulation of covariant Schrödinger dynamics of a single particle, we present here the case for the consideration of a Hilbert space for state vectors with an indefinite norm. The key notion is the noncompact nature of the Lorentz group $S O(1,3)$ giving all finite dimensional representations as non-unitary, hence failing to preserve any positive definite norm on the representation space. But it is the finite dimensional representations that serve as the natural extensions of the corresponding unitary ones of the $S O(3)$ rotational subgroup. In particular, the Minkowski spacetime as a representation space of $S O(1,3)$ is $(1+3)$-dimensional splitting into the single time space and the 3-dimensional space in the Newtonian limit. Minkowski spacetime, of course, has an invariant inner product on which a Lorentz boost acts as a non-unitary transformation while a rotation acts as a unitary one. It is exactly the kind of pseudo-unitarity we suggest to be incorporated as a basic structure of a fully Lorentz covariant quantum mechanics.

\section{The Covariant Harmonic Oscillator}

The kind of quantum theory we have in mind can easily be appreciated in the covariant harmonic oscillator problem, which has been among the first studies of a Lorentz covariant quantum mechanics. It is important to note that the problem actually goes beyond the setting of Poincaré symmetry. The proper symmetry behind the problem is that of $H_{R}(1,3)$ given as

$$
\begin{aligned}
& {\left[J_{\mu \nu}, J_{\rho \sigma}\right]=i \hbar\left(\eta_{v \sigma} J_{\mu \rho}+\eta_{\mu \rho} J_{v \sigma}-\eta_{\mu \sigma} J_{v \rho}-\eta_{v \rho} J_{\mu \sigma}\right),} \\
& {\left[J_{\mu \nu}, X_{\rho}\right]=i \hbar\left(\eta_{\mu \rho} X_{v}-\eta_{v \rho} X_{\mu}\right),} \\
& {\left[J_{\mu \nu}, P_{\rho}\right]=i \hbar\left(\eta_{\mu \rho} P_{v}-\eta_{\nu \rho} P_{\mu}\right),} \\
& {\left[X_{\mu}, P_{v}\right]=i \hbar \eta_{\mu v} I,}
\end{aligned}
$$

where we have adopted $\eta_{\mu v}=\operatorname{diag}\{-1,1,1,1\}$. Naively, one wants to think about the operator representation with $\hat{X}_{\mu}$ given by $x_{\mu}, \hat{P}_{\mu}$ by $-i \hbar \frac{\partial}{\partial x^{\mu}}$, $\hat{J}_{\mu \nu}=\hat{X}_{\mu} \hat{P}_{v}-\hat{X}_{v} \hat{P}_{\mu}$, while $I$ represented by the identity operator. The representation is unitary and does not work so well as the case of the familiar $H_{R}(3)$ 
setting at all [3]. In fact, unitarity and Lorentz covariance together would force taking only Lorentz invariant states as admissible, while on the technical side the wavefunctions and the integral norm have divergence issues. We emphasize the perspective here of having a formulation and solutions as a natural extension of the $H_{R}(3) \equiv H_{R}(0,3)$ case without divergence problems. The noncompact nature of $S O(1,3)$ generated by the $J_{\mu v}$ then points towards its pseudo-unitary representations.

There is a parallel problem for any $H_{R}(l, m)$. The Hermitian operator $\hat{N}$ representing $\frac{1}{2 \hbar}\left(X_{\mu} X^{\mu}+P_{\mu} P^{\mu}\right)$, plus a constant, commutes with all $\hat{J}_{\mu \nu}$. Each fixed $n$-level, for $n$ being the eigenvalue of $\hat{N}$, corresponds to a representation of $S O(l, m)$. We want the $n=0$ level to be the trivial representation and the $n=1$ level to be the defining vector representation. The latter is to say, the real span of $n=1$ Fock states is essentially a $(l+m)$-dimensional pseudo-Euclidean space of signature $(l, m)$. The higher $n$ levels then naturally correspond to symmetric Cartesian/pseudo-Euclidean tensors each of which splits into irreducible representations of $S O(l, m)$ corresponding to the rank of the tensors. Of course, all such representations at any finite $n$ are finite dimensional and non-unitary. The $S O(l, m)$ transformations are to be represented by "rotations" on the pseudo-Euclidean space preserving the pseudo-Euclidean inner product. The Hilbert space as the space spanned by all Fock states can be seen as the natural complex extension of it. We will soon report on a detailed analysis with explicit Fock state wavefunctions and the pseudo-unitary inner product along the line.

\section{Theory from Symmetry Representation and the Geometric Picture}

Basic quantum mechanics is really a representation theory of group $H_{R}(3)$ [4], of which $H_{R}(1,3)$ is a natural Lorentz covariant extension. In the former case, a natural representation to use is an irreducible component of the regular representation of the Heisenberg-Weyl symmetry $H(3)$, all of which can be seen as essentially giving the same physics. Such representations are spin zero representations of the full $H_{R}(3)$ with $J_{i j}$ given by $X_{i} P_{j}-X_{j} P_{i}$. The central charge $I$ has to be represented by a multiple of an identity. Taking the latter as a positive real number $\zeta$ times the identity $\hat{I}$, we have $\left[\hat{X}_{\zeta i}, \hat{P}_{\zeta j}\right]=i(\zeta \hbar) \delta_{i j} \hat{I}$. We should then identify the true physical position and momentum operators as $\frac{1}{\sqrt{\zeta}} \hat{X}_{\zeta i}$ and $\frac{1}{\sqrt{\zeta}} \hat{P}_{\zeta i}$. For a representation with a negative $\zeta$, we should identify $\frac{1}{\sqrt{|\zeta|}} \hat{P}_{\zeta i}$ as the position operators $\hat{X}_{i}$ and $\frac{1}{\sqrt{|\zeta|}} \hat{X}_{\zeta i}$ as the momentum operators $\hat{P}_{i}$. The regular representation can be expressed as a direct integral of the irreducible components for all real values of $\zeta$, with a measure vanishing at the $\zeta=0$ point, which does not correspond to one such component [5]. Each 
component then has the natural description with observables given by $\alpha\left(p^{i} \star, x^{i} \star\right)=\alpha\left(p^{i}, x^{i}\right) \star$, like functions of the position and momentum operators $x^{i} \star$ and $p^{i} \star$, as operators acting on the states with wave functions $\phi\left(p^{i}, x^{i}\right)=\left\langle p^{i}, x^{i} \mid \phi\right\rangle$, where $\left|p^{i}, x^{i}\right\rangle$ are the coherent states and $\star$ is the "product" corresponding to the Moyal star-product of $\alpha \star \beta$ [4].

One lesson from above is that there is no need at all to think about a negative effective $\hbar$ value. We have one theory of quantum mechanics the one particle phase space of which is a Hilbert space for one value of $\zeta$, for which we know $\left[\hat{X}_{i}, \hat{P}_{j}\right]=i \hbar \delta_{i j} \hat{I}$. Moreover, the free particle phase space can be seen as the proper quantum model of the physical space on which quantum mechanics is the associated symplectic mechanics. Under the proper formulation, the physical space model and the dynamical theory reduce back exactly to the Newtonian ones at the classical limit [4] [6]. The perspective matches with the intuitive idea that the physical space is the collection of all possible positions for the particle. That is to say, only the single representation with the observed $\hbar$ value is physically relevant.

The situation is however different in the case of $H_{R}(1,3) . H(1,3)$ and $H(4)$ are isomorphic, i.e. really the same so long as we do not have a priori identification of the generators with physical observables. $H_{R}(1,3)$ and $H_{R}(4)$ are definitely different as (real) Lie groups/algebras though. The relative sign in $\eta_{\mu \nu}$ says that the $X_{0}-P_{0}$ pair maintaining the mathematical nature as the components of the $X_{\mu}-P_{\mu}$ four-vectors has a commutator of a different sign from the $X_{i}-P_{i}$ pairs, which has to be preserved in an representation of $H_{R}(1,3)$ with the position and momentum operators being Minkowski four-vectors. Hence, we cannot avoid having the commutator $\left[X_{0}, P_{0}\right]$, or actually in terms of the corresponding operators in the physical representation $\left[\hat{X}_{0}, \hat{P}_{0}\right]$, being equal to $-i \hbar \hat{I}$, analogous to an effective $\hbar$ value being negative.

Quantum mechanics can completely be described by the symplectic or Kähler geometry of its phase space, the infinite dimensional projective Hilbert space. The observable algebra corresponds to an algebra of the so-called Kählerian functions and Schrödinger dynamics is given by their Hamiltonian flows [7]. Most importantly, $\hbar$, or its effective value, the real parameter in the commutator $[\hat{X}, \hat{P}]$, characterizes the constant holomorphic sectional curvature of the Kähler geometry. In any $H_{R}(n)$ setting then, the projective Hilbert space is compact and positively curved. A natural conclusion is that for a theory with an effective negative value of the commutator, the corresponding phase space would have a negative curvature. Kawamura [8] has indeed discussed such a case as a plausible generalization of quantum mechanics, unfortunately without drawing any connection to the relevant $S O(m, n)$ symmetry. Mathematically, the compact projective Hilbert space can be seen as a coset space $S U(N) / S U(N-1)$ with $N$ taken to the infinite limit. The noncompact negatively curved analogs are given by $S U(M, N) / S U(M, N-1)$ or $S U(M, N) / S U(M-1, N)$, from a Hilbert space with a $S U(M, N)$ invariant pseudo-unitary inner product [9]. 


\section{Final Remarks}

It is important to note that the covariant harmonic oscillator problem and the formulation of the quantum mechanics itself are much the same. For the usual quantum mechanics, as the unitary representation of $H_{R}(3)$ with Hermitian position and momentum operators, for example, the true Hilbert space is not that of the square-integrable functions even for the $\phi\left(x^{i}\right)$ wavefunction formulation. It is a dense subspace of rapidly decreasing functions, the most ready explicit picture of which is the span of the harmonic oscillator Fock states [10]. Recall that the coherent states can be constructed from the Fock states too. Our analysis points above are in the same direction of pseudo-unitary representation.

We have mentioned above that the projective Hilbert space should be seen as the proper model of the physical space behind quantum mechanics, from which one can retrieve the correct classical limit. It is also true that the submanifold of the coherent states is exactly like a copy of the classical phase space sitting inside the quantum one. The classical phase space is naively a simple product of the space/configuration part and the momentum part with the same Euclidean geometry. In fact, their metrics are simply given by restrictions of the metric for the projective Hilbert space [11]. When one goes to the Lorentz covariant case, the corresponding coherent state submanifold obviously needs to have a metric of Minkowski signature for the spacetime/configuration part and the momentum part. The latter obviously asks for a metric or inner product with an indefinite signature for the quantum Hilbert space. We hope to report on an explicit formulation of such a quantum theory in the near future.

\section{Acknowledgements}

Special thanks go to Suzana Bedi'c for discussions and assistance in editing the manuscript, as well as collaboration on related studies. The author is partially supported by research grant number 107-2119-M-008-011 of the MOST of Taiwan.

\section{Conflicts of Interest}

The authors declare no conflicts of interest regarding the publication of this paper.

\section{References}

[1] Kong, O.C.W. (2019) An Intuitive Geometric Picture of Quantum Mechanics with Noncommutative Values for Observables NCU-HEP-k081.

[2] Kong, O.C.W. (2019) Quantum Spacetime Pictures and Particle Dynamics from a Relativity Perspective. AIP Conference Proceedings, 2075, 100001, 10pp. https://doi.org/10.1063/1.5091245

[3] Bars, I. (2009) Relativistic Harmonic Oscillator Revisited. Physical Review D, 79, 045009. https://doi.org/10.1103/PhysRevD.79.045009

[4] Chew, C.S., Kong, O.C.W. and Payne, J. (2019) Observables and Dynamics, Quantum to Classical, from a Relativity Symmetry Perspective. Journal of High Energy 
Physics, Gravitation and Cosmology, 5, 553-586.

https://doi.org/10.4236/jhepgc.2019.53031

[5] Taylor, M.E. (1986) Noncommutative Harmonic Analysis. American Mathematical Society, Providence, Rhode Island. https://doi.org/10.1090/surv/022

[6] Chew, C.S., Kong, O.C.W. and Payne, J. (2017) A Quantum Space Behind Simple Quantum Mechanics. Advances in High Energy Physics, 2017, Article ID: 4395918. https://doi.org/10.1155/2017/4395918

[7] Cirelli, R., Manià, A. and Pizzocchero L. (1990) Quantum Mechanics as an Infinite-dimensional Hamiltonian System with Uncertainty Structure: Part I. Journal of Mathematical Physics, 31, 2891-2897. https://doi.org/10.1063/1.528941

[8] Kawamura, K. (1997) Quantum Mechanics and Operator Algebras on the Hilbert Ball, arXiv. funct-an/9710002.

[9] Boothby, W.M. and Weiss G.L. (1972) Symmetric Spaces, Marcel Dekker, Inc.

[10] Dubin, D.A., Hennings, M.A. and Smith T.B. (2000) Mathematical Aspects of Weyl Quantization and Phase. World Scientific, Singapore. https://doi.org/10.1142/4140

[11] Bengtsson, I. and Życzkowski, K. (2006) Geometry of Quantum States. Cambridge University Press, Cambridge. https://doi.org/10.1017/CBO9780511535048 